\title{
Nucleotide Sequence of Messenger RNA Encoding Human Isovaleryl-Coenzyme A Dehydrogenase and Its Expression in Isovaleric Acidemia Fibroblasts
}

Yoichi Matsubara, Michinori lto, Robin Glassberg, Sundermoorthy Satyabhama, Yasuyuki Ikeda, and Kay Tanaka

Department of Human Genetics, Yale University School of Medicine, New Haven, Connecticut 06510

\begin{abstract}
Isovaleric acidemia (IVA) is caused by a genetic deficiency of isovaleryl-CoA dehydrogenase (IVD). At least five distinct variant IVD alleles are known. We isolated five overlapping IVD cDNA clones from a human placenta cDNA library. They covered the entire coding region, except the initiation codon, and $587 \mathrm{bp}$ in the $3^{\prime}$-noncoding region plus the poly(A) tail. The structure of the initiation site was identified by the study of genomic DNA and by the sequence comparison with rat IVD. Human IVD shared 89.6, 35.8, and 31.6\% identical amino acid residues with rat IVD and human short and medium chain acyl-CoA dehydrogenases, respectively. In the Northern blot analysis of normal human liver and fibroblast poly $(A)^{+}$RNA, three mRNA species of different sizes $(4.6,3.8$, and $2.1 \mathrm{~kb})$ hybridized to IVD cDNA. Three mRNA species with similar sizes were also detected in five IVA fibroblast lines of different genotypes (variants $1,1 \times 2,2,3$, and 5), suggesting that these variants are each due to a point mutation or small deletion. ( $J$. Clin. Invest. 1990. 85:1058-1064.) isovaleryl-CoA dehydrogenase $\bullet$ isovaleric acidemia $\bullet$ messenger RNA $~$ cDNA • variant
\end{abstract}

\section{Introduction}

Isovaleric acidemia (IVA) ${ }^{1}$ is an autosomal-recessively inherited disorder caused by a deficiency of isovaleryl-CoA dehydrogenase (IVD; EC 1.3.99.10) $(1,2)$. IVD catalyzes the third reaction in leucine metabolism. IVD is one of the five enzymes that belong to the acyl-CoA dehydrogenase family (3). Other enzymes in this group are short chain (SCAD), medium chain

Portions of this work were published in abstract form (1988. Pediatr. Res. 23:322A. [Abstr.]).

Dr. Matsubara's present address is Tohoku University School of Medicine, Departments of Pediatrics and Biochemical Genetics, 1-1 Seiryo-machi, Sendai 980, Japan. Dr. Ito's present address is Tokushima University, School of Medicine, Department of Pediatrics, 3 Kuramoto-cho, Tokushima 770, Japan. Dr. Ikeda's present address is National Cardiovascular Center Research Institute, 5-125 Fujishirodai, Suita 565, Osaka, Japan.

Address reprint requests to Dr. Kay Tanaka, Yale University School of Medicine, Department of Human Genetics, 333 Cedar Street, P. O. Box 3333, New Haven, CT 06510.

Received for publication 23 August 1989 and in revised form 29 November 1989.

1. Abbreviations used in this paper: $\alpha$-ETF, the $\alpha$-subunit of electron transfer flavoprotein; IVA, isovaleric acidemia; IVD, isovaleryl-CoA dehydrogenase; LCAD, long chain acyl-CoA dehydrogenase; MCAD, medium chain acyl-CoA dehydrogenase; $n t$, nucleotide; $p$, precursor; SCAD, short chain acyl-CoA dehydrogenase.

J. Clin. Invest.

(c) The American Society for Clinical Investigation, Inc.

0021-9738/90/04/1058/07 \$2.00

Volume 85, April 1990, 1058-1064
(MCAD), long chain (LCAD), and 2-methyl-branched chain acyl-CoA dehydrogenases. 2-Methyl-branched chain acyl-CoA dehydrogenase is involved in the metabolism of isoleucine and valine, whereas the three others catalyze the first step of the $\beta$-oxidation cycles for fatty acids with various chain length (4-7). IVD was first identified and purified to homogeneity from rat liver by Ikeda and Tanaka in $1983(4,5)$ and extensively characterized along with four other homologous acylCoA dehydrogenases $(4,6,7)$. Subsequently, IVD was also purified to homogeneity from human liver (8). Like four other acyl-CoA dehydrogenases, IVD is a flavin adenine dinucleoside-containing mitochondrial matrix enzyme consisting of four identical subunits. The sizes of human and rat IVD were both estimated to be $43 \mathrm{kD}$. Like most mitochondrial enzymes, IVD is synthesized in the cytosol as a precursor (p) with an extra leader peptide, imported into mitochondria and proteolytically processed into the mature form (9). The sizes of both human and rat pIVD have been estimated to be $45 \mathrm{kD}$ $(9,10)$.

The clinical symptoms of IVA are episodic vomiting, lethargy, and coma, which are accompanied by a peculiar sweaty feet odor $(1,11,12)$. Episodic accumulation of isovaleric acid in blood and continuous excretion of its glycine conjugate in urine are the major biochemical findings. Two clinical phenotypes, acute and chronic intermittent forms, have been noted (12). However, close scrutiny of the history of individual cases revealed that the distinction of the two clinical phenotypes is arbitrary and unclear. Furthermore, the accurate assay of IVD activity in cultured skin fibroblasts from nine IVA patients, including both types, indicated that all of the cell lines had uniformly low IVD activity regardless of the clinical phenotype (13).

In the previous study of 12 IVA cell lines using polyethylene glycol-induced heterokaryons, no complementation groups were found (14), indicating the involvement of a single IVD gene in this disease. However, the analysis of variant IVD in 15 IVA fibroblast cell lines using $\left[{ }^{35}\right.$ S $]$ methionine labeling, immunoprecipitation, and SDS-PAGE revealed five distinct classes of variants (10). The molecular size of variant 1 was indistinguishable from their normal counterparts in both the precursor and mature forms (45 and $43 \mathrm{kD}$, respectively), although the activity of this variant was as deficient as that of any other variant (12). Variant 2 was synthesized as a truncated $(42-\mathrm{kD})$ precursor, but only a small portion of it was processed to the $40-\mathrm{kD}$ mature form. Variants 3 and 4 were also synthesized each as a truncated precursor ( 43 and $42 \mathrm{kD}$, respectively), and both of them were efficiently processed to the $41-$ and $40-\mathrm{kD}$ mature forms, respectively. In variant 5 cells, no immunoprecipitable IVD was detectable (10).

We have recently reported the isolation and sequence of rat liver IVD cDNA clones and assigned the IVD gene to human chromosome $15(3,15)$. We now report here the isolation and nucleotide sequence of human IVD cDNAs and the study of IVD mRNA in four different types of IVA cell lines using the rat IVD $\mathrm{CDNA}$ as a probe. 


\section{Methods}

Materials. IVA cell lines used in this study were No. 501 (variant 1), No. 834 (variants $1 \times 2$ ), No. 747 (variant 2), No. 765 (variant 3), and No. 1339 (variant 5). Sources of the cells were previously reported (10). Restriction enzymes were from New England Biolabs (Beverly, MA).

Screening of human placenta cDNA library. A near-full length rat IVD cDNA (rpIVD-17) was radiolabeled by the random primer DNA-labeling method (16) and used as a probe to screen $9 \times 10^{5}$ plaques from a human placenta cDNA library (Clonetech Laboratories, Palo Alto, CA) that contained $1 \times 10^{6}$ independent recombinants. Hybridizations were carried out at $65^{\circ} \mathrm{C}$ in $6 \times$ SSPE, $5 \times$ Denhardt's solution, $0.5 \%$ SDS, $5 \mathrm{mM}$ 2-[Tris(hydroxymethyl)methylamino]-1ethanesulfonic acid, and $10 \mu \mathrm{g} / \mathrm{ml}$ salmon sperm DNA. The hybridized nitrocellulose filters were washed twice with $6 \times$ SSPE, $0.1 \%$ SDS at $65^{\circ} \mathrm{C}$ for $30 \mathrm{~min}$.

DNA sequencing. Restriction fragments of IVD cDNAs were subcloned into pGEM-blue plasmid (Promega Biotec, Madison, WI). Nucleotide sequence was determined directly from double-stranded DNA (17) using the dideoxy-sequencing method (18). To eliminate sequencing ambiguities, the entire nucleotide sequence was sequenced twice, once using Klenow fragment of Escherichia coli DNA polymerase I (Boehringer Mannheim Biochemicals, Indianapolis, IN) and the second time using Sequenase (United States Biochemical Corp., Cleveland, $\mathrm{OH}$ ). In some experiments dGTP was replaced with 7 deaza-dGTP or dITP to avoid ambiguity due to GC compression.

Cloning and sequencing of genomic DNA encoding IVD. A sorted human chromosome 15 genomic DNA library (American Type Culture Collection, Rockville, MD) was screened for IVD gene-related DNA sequences by the plaque hybridization method $(19,20)$ using ${ }^{32}$ P-labeled human IVD-cDNA (hpIVD-8) as a probe. The library contained complete Hind III digests with the nominal average size of 4 $\mathrm{kb}$ in the $\lambda$-based vector, Charon $21 \mathrm{~A}$. The yield of independent recombinants was $7 \times 10^{4}$, with the number of chromosome equivalents of the amplified library being 4.4. Approximately $3.5 \times 10^{5}$ plaques were screened. Plaque dilution and DNA purification from the positive genomic clones were followed by restriction endonuclease digestion and Southern blot analysis using hpIVD-8 as probe. The isolated DNA fragments were subcloned into Bluescript plasmid (Stratagene; San Diego, CA) and sequenced.

Computer analysis of nucleotide sequence. The computer programs of the University of Wisconsin Genetics Computer Group (21) were used to align or compare nucleotide sequence data.

Northern blot analysis. Total RNAs were isolated from rat and postmortem human livers, and cultured human fibroblasts were obtained from a normal individual and IVA patients according to the method of Chirgwin et al. (22). RNAs were then applied to an oligo-dT column to obtain poly(A) ${ }^{+}$RNAs. Poly(A) ${ }^{+}$RNAs were electrophoresed in $2.2 \mathrm{M}$ formamide/1\% agarose gel, transferred to a nitrocellu- lose filter (Schleicher \& Schuell, Inc., Keene, NH), and hybridized with radiolabeled IVD cDNA (hpIVD-8) or a rat IVD CDNA, rpIVD-A3 (3, 15). After thoroughly stripping the IVD probe, the same filter was hybridized with a cDNA encoding the $\alpha$-subunit of electron transfer flavoprotein $(\alpha$-ETF) (E9f), which was previously isolated and sequenced in our laboratory (23).

\section{Results}

Isolation of cDNAs encoding human placenta IVD. Using radiolabeled 1.1-kb rat IVD cDNA, rpIVD-17 $(3,15)$, as a probe, $9 \times 10^{5}$ plaques from a human placenta cDNA library were screened. Five positive clones (hpIVD-1, -3, -5, -6, and -8) were obtained. The length of the cDNA inserts of these positive clones ranged from 900 (hpIVD-3) to $1,800 \mathrm{bp}$ (hpIVD-8), overlapping with each other as tested by restriction enzyme mapping (Fig. 1). cDNA inserts from all five clones were excised with Eco RI and subcloned into pGEM-blue plasmids. The nucleotide sequences of hpIVD-5 and -8 were determined in both directions according to the sequence strategy as shown in Fig. 1.

Nucleotide and amino acid sequences of human IVD. Among the human IVD cDNA clones isolated, hpIVD-8 and hpIVD-5 extended farthest toward the 5 '- and 3 '-ends, respectively (Fig. 1). Both of them were $\sim 1,800$ bp long, overlapping greatly. When combined, these two cDNA inserts covered a region of $1,883 \mathrm{bp}$, including a 1,266 -bp coding region. Comparison of these sequences to that of rat pIVD cDNA revealed a high degree of homology (see below), confirming that the isolated cDNA clones indeed encoded human pIVD. However, neither hpIVD-8 nor hpIVD-5 contained the initiation codon. The Ala, which is encoded by the GCG triplet at the $5^{\prime}$ end of hpIVD-8, was considered to be the penultimate amino terminal residue as in the case of rat pIVD. The 18 residues immediately following this Ala in human and rat pIVD sequences matched well (3). In this 18 amino acid segment of human and rat pIVD sequences, only three amino acid residues, Thr-4, Trp-9, and Ala-12, were discordant.

The sequence at the $5^{\prime}$ end of the coding region and in the immediately preceding 5 '-flanking region was studied using genomic DNA. Five overlapping clones that appeared to cover the entire length of the IVD gene were obtained. Two of them ( 3 and $6.5 \mathrm{~kb}$ ) contained this region. The GCG codon, encoding the first Ala residue in hpIVD-8, was indeed next to an ATG codon, which corresponded to the initiation codon of rat pIVD. Unexpectedly, however, another in-frame ATG was
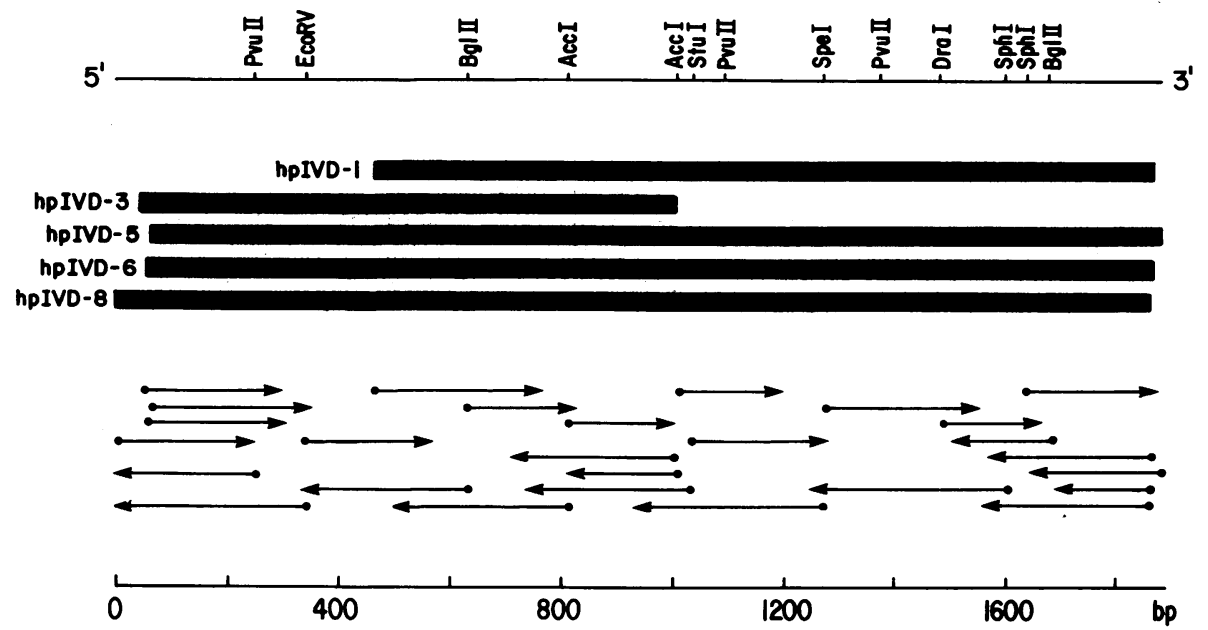

Figure 1. Partial restriction map of human pIVD cDNA clones and sequencing strategy. The arrows indicate the direction and extent of sequencing. 
found nine nucleotides (nt) upstream. The entire nucleotide and deduced amino acid sequences of human IVD cDNA, supplemented with the information from the genomic DNA study, are shown in Fig. 2. The downstream ATG was assigned to be the initiation codon, and the nucleotide and amino acid positions were counted taking the adenine in the downstream ATG codon and the corresponding Met as the first residues, respectively, in these sequences for the following reasons. First, the downstream ATG codon is preceded $3 \mathrm{nt}$ upstream by a purine (G). Also, it is immediately followed by a guanine (Fig. 3). This pattern conforms with the eukaryotic consensus sequence for the initiation site $(24,25)$. In contrast, the upstream ATG is preceded by a pyrimidine (T) three nt upstream. The latter pattern is frequently seen in nonfunctional ATG triplets that lie upstream of the initiation site $(24,25)$. Second, when the nucleotide sequences of human and rat pIVD cDNA immediately adjacent the initiation site are compared, the downstream ATG in the human pIVD cDNA can be readily aligned with the rat initiation codon, while the upstream ATG cannot (Fig. 3). Third, if the upstream ATG is functional, the third amino acid residue would be Glu. In general, acidic residues are rare in the leader peptide of nuclear coded mitochondrial enzymes $(26,27)$.

When counted from the downstream ATG, the coding region of human pIVD precursor is $1,269 \mathrm{bp}$, that is, 3 bases shorter than rat pIVD. The amino terminus of the mature human IVD has not been determined. The amino terminus of rat IVD is His-30. Comparison of the nucleotide sequences of human and rat pIVD cDNAs indicates that the human sequences upstream of $\mathrm{C}-56$ and downstream of $\mathrm{C}-78$ are very similar to the regions of rat sequences upstream of C-56 and downstream of C-82, respectively. In these regions, their sequences usually match residue to residue, with the identical total number of residues. In comparison, the sequences in the section between C-56 and C-78 (C-82 in the rat sequence) are very dissimilar. Thus, the 3-bp deletion in human pIVD occurred in the leader sequence somewhere between C-56 and G-81. Counted from the downstream ATG and calculated on the assumption that the amino terminus in the human mature IVD is the same as in rat IVD, the molecular sizes of the precursor, mature IVD and leader peptide are 46,325, 43,074, and 3,269 , respectively. The 29 amino acid leader peptide contains five Arg residues and no acidic amino acid residues. Thus, the leader peptide of human pIVD is overall positively charged, as in the case of any other nuclear coded mitochondrial matrix enzyme $(26,27)$.

hpIVD-3 contains a 597-bp 3 '-noncoding region and a poly(A) tail that is preceded $21 \mathrm{nt}$ upstream by a consensus signal for polyadenylation (AATAAA). It is interesting to note that there is a repeat of a 38 -residue sequence in the 3 'snoncoding region $(1,572-1,609$ and $1,610-1,647)$. This repeat is particularly rich in $\mathrm{GC}$ residues $(27$ of $38 ; 71 \%)$. Furthermore, an octanucleotide sequence, CCCACCTC, that is present in the beginning part of each of the 38-nt repeats, is repeated once more immediately after the second 38-nt repeat. These repeated sequences are not cloning artifacts, because the same repeats were identified in the genomic IVD DNA (Satyabhama, S., and K. Tanaka, unpublished observations).

Sequence comparison to rat IVD and human SCAD and $M C A D$. Homology of the entire coding region of human and rat pIVD cDNAs is $87 \%$ at the nucleotide level. At the amino acid level, human and rat pIVD share $89.6 \%$ identical residues as shown in Fig. 4. When conservative substitutions are in- cluded, the sequence similarity is $96.2 \% .^{2}$ In the leader peptide domain human and rat pIVD share only $67 \%$ identical amino acid residues, while they share $91.4 \%$ identical residues in the mature enzyme domain. In the mature enzyme domain, sequence similarity is lower in the first 50 residues of the aminoterminal region, with $78 \%$ identical residues, than in the remaining portion in which $93.3 \%$ identical residues are shared.

Human pIVD share with human pSCAD (28) and pMCAD (29) 35.8 and $31.6 \%^{2}$ identical residues, respectively. When conservative substitutions are included, the sequence similarity between human pIVD and the two other enzymes are 54.5 and $56.0 \%{ }^{2}$ Alignment of human pIVD and pSCAD is shown in Fig. 5. Several sections with relatively low similarity are scattered.

Analysis of $m R N A$ from normal human and rat livers. To study pIVD mRNA, a poly(A) ${ }^{+}$fraction was isolated from human and rat total liver RNAs, then electrophoresed and blotted (Fig. 6). When hybridized with the radioactive rat pIVD cDNA (rpIVD-A3), three radioactive bands (4.6, 3.8, and $2.1 \mathrm{~kb}$ ) were observed in human liver mRNA (lane 2). The 2.1-kb mRNA matches the size of cDNA and therefore is considered to be the translatable mRNA species. The size of rat liver IVD mRNA was estimated to be $2.4 \mathrm{~kb}$ (lane 4 ). When the Northern blot filter for rat RNA was exposed to $x$-ray film for a longer period of time (lane 3), a sharp minor mRNA band of larger size $(4.8 \mathrm{~kb})$ became visible as in the case of human IVD. This minor band did not disappear even after stringent washing of the filter $\left(0.1 \times \mathrm{SSPE}, 65^{\circ} \mathrm{C}, 1 \mathrm{~h}\right)$.

Analysis of $m R N A$ from cultured normal and IVA fibroblasts. As in the case of human liver mRNA, three bands of $4.6,3.8$, and $2.1 \mathrm{~kb}$ were identified in the Northern blot hybridization analysis of RNAs from normal human cultured fibroblast cell lines (Fig. 7, lane 1). The fourth 1.6-kb band was $\mathrm{p} \alpha$-ETF mRNA. The p $\alpha$-ETF probe was hybridized with the same filter after the pIVD probe was thoroughly stripped, and its $x$-ray film was accurately overlaid with that for pIVD, so that the $\alpha$-ETF mRNA band could be used as an internal standard for accurately defining the degree of migration of pIVD mRNA bands in each cell extract. The patterns of Northern blot analysis of pIVD mRNA from four different types of IVA cells, including variants $1,2,3$, and 5, were similar to those in the normal fibroblasts, each exhibiting three bands with the sizes that are similar to their normal counterparts (Fig. 7, lanes 2-6). The detection of mRNAs with normal size in variant 5 cells was unexpected because this variant was crossreactive materials-negative. Variant $4 \mathrm{mRNAs}$ could not be analyzed this time since the only cell line of this type was lost after our initial study.

\section{Discussion}

All five acyl-CoA dehydrogenases catalyze $\alpha, \beta$-dehydrogenation of acyl-CoA esters and transfer electrons to ETF (30) via the same mechanism (31). However, they distinctly differ in the length and configuration of the hydrocarbon chain of the substrate $(4-7,32)$. For the physical and functional similarities that they share, we had previously proposed that the acyl-CoA dehydrogenases belong to a gene family, the acyl-CoA dehy-

2. In the calculations of sequence similarity, the number of identical amino acid residues, shared by two proteins, was divided by the larger of the two numbers of the total amino acid residues in the pertinent proteins. 


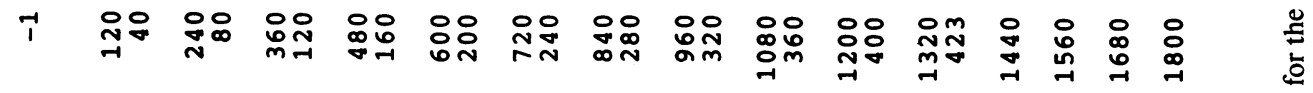

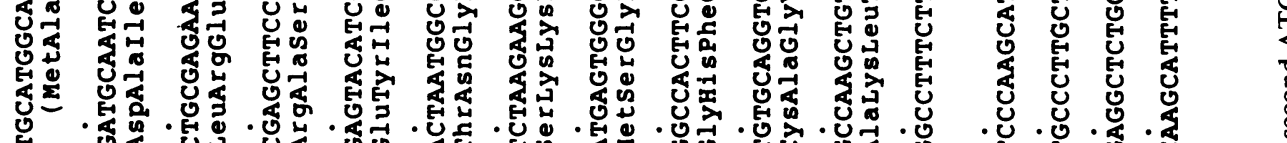

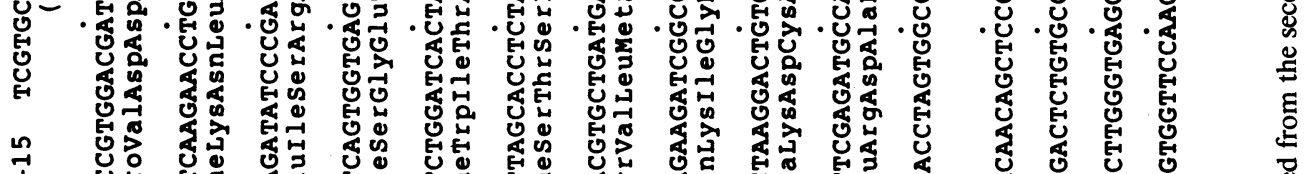

1

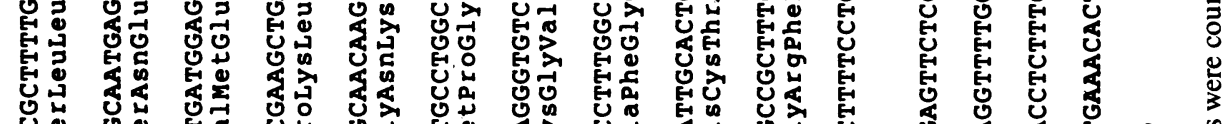

.

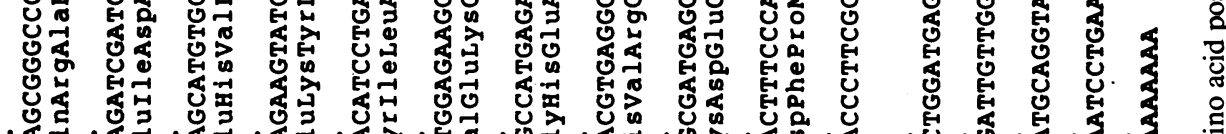

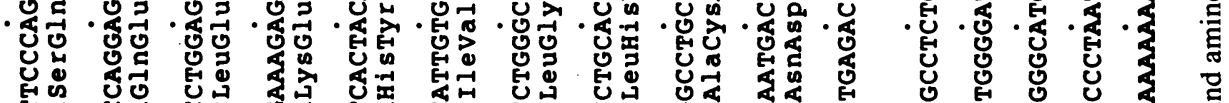

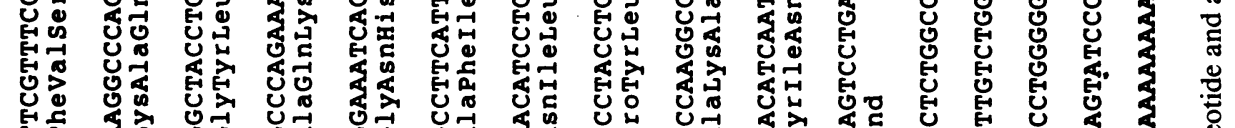

.

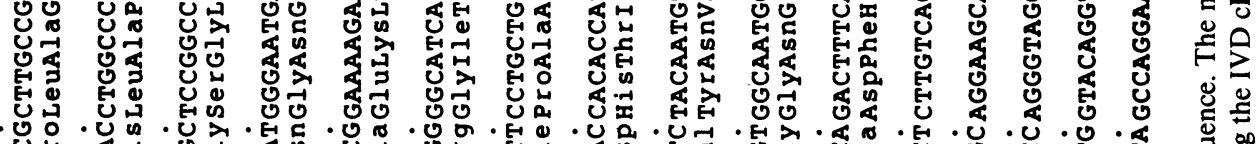

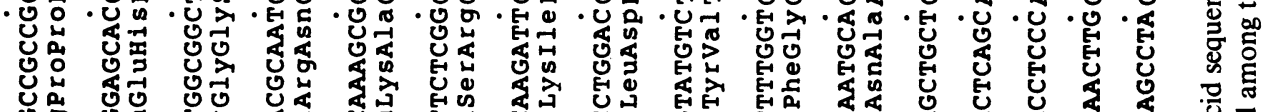

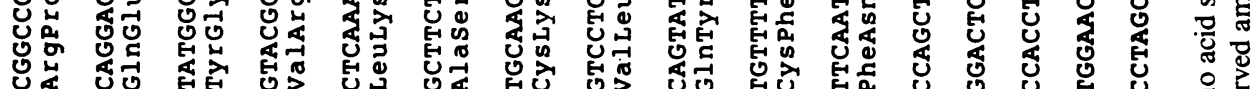

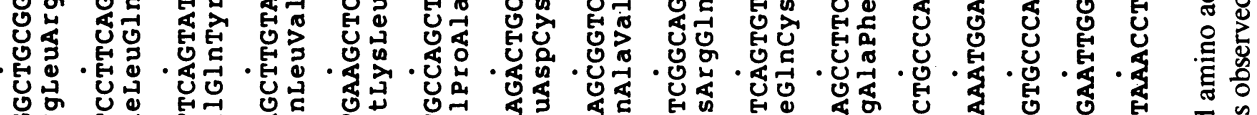

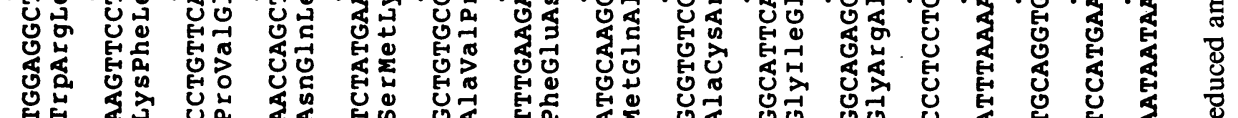

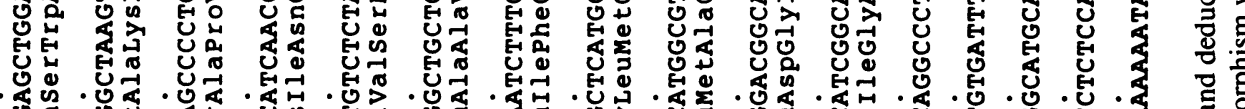

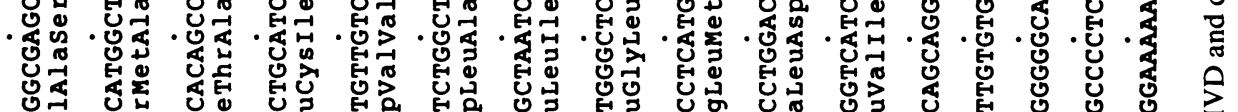

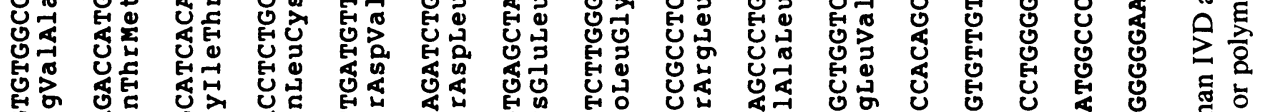

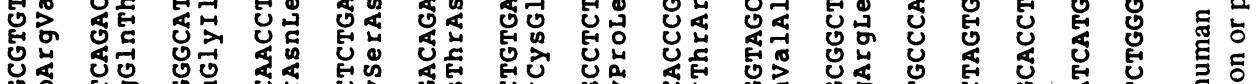

.

H:

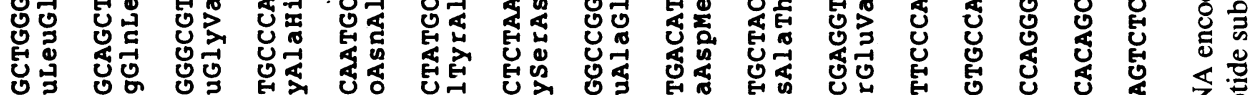

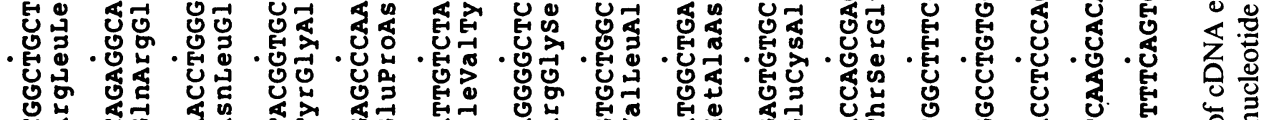

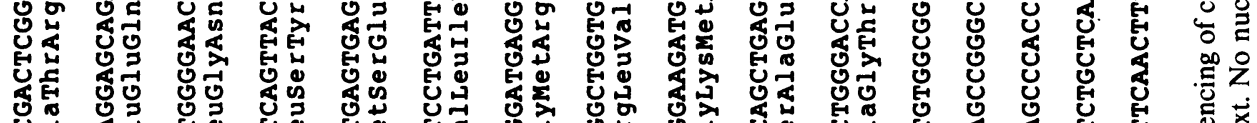

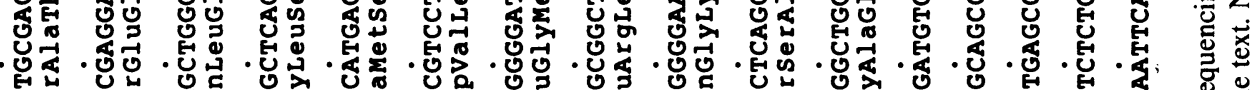

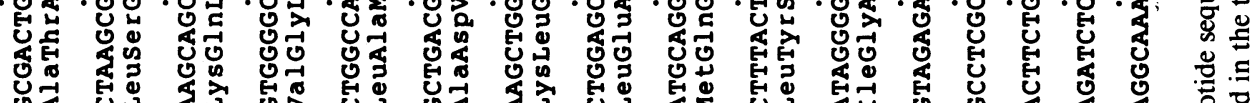

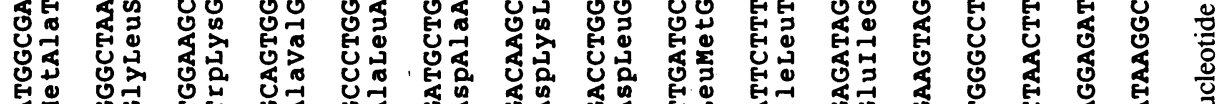

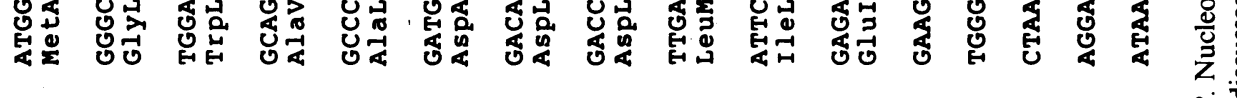

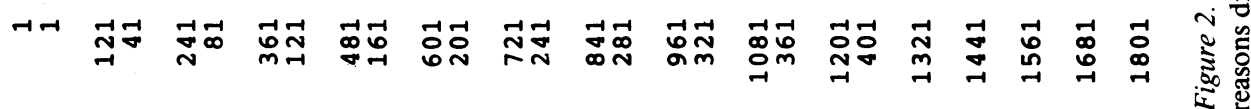


HIVD

RIVD

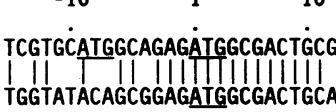

HIVD

RIVD nucleotide sequences around the initiation site of the human and rat pIVD. The initiation codon $(A T G)$ of rat pIVD was aligned with the downstream ATG $(A)$ and with the upstream ATG $(B)$ of human pIVD.
Figure 3. Alignments of the

drogenase family, and evolved from a common ancestral gene (32). Recently, we have cloned and sequenced cDNAs, each encoding rat liver MCAD (33, 34), LCAD, SCAD, IVD (3), and human SCAD (28). The sequences of these enzymes, together with that of human MCAD sequenced by Kelly and associates (29) provided molecular evidence that these acylCoA dehydrogenases indeed belong to a gene family (3). The addition of human IVD to this list provides further information in this regard.

The number of identical residues shared by human pIVD and pSCAD was slightly higher than those shared by pIVD and pMCAD. The same relationship has been observed among their rat counterparts. Thus, the evolutionary distance between pIVD and pSCAD is closer than that between pIVD and pMCAD. The degree of sequence similarity between human and rat pIVD is of different magnitude, sharing close to $90 \%$ identical residues. The number of consecutive identical residues is naturally much higher between two pIVD from the two different species than between two different acyl-CoA dehydrogenases from the same species. The number of consecutive identical residues shared by human and rat pIVD are often $>15$, with the highest being 33 . In contrast, the highest num-

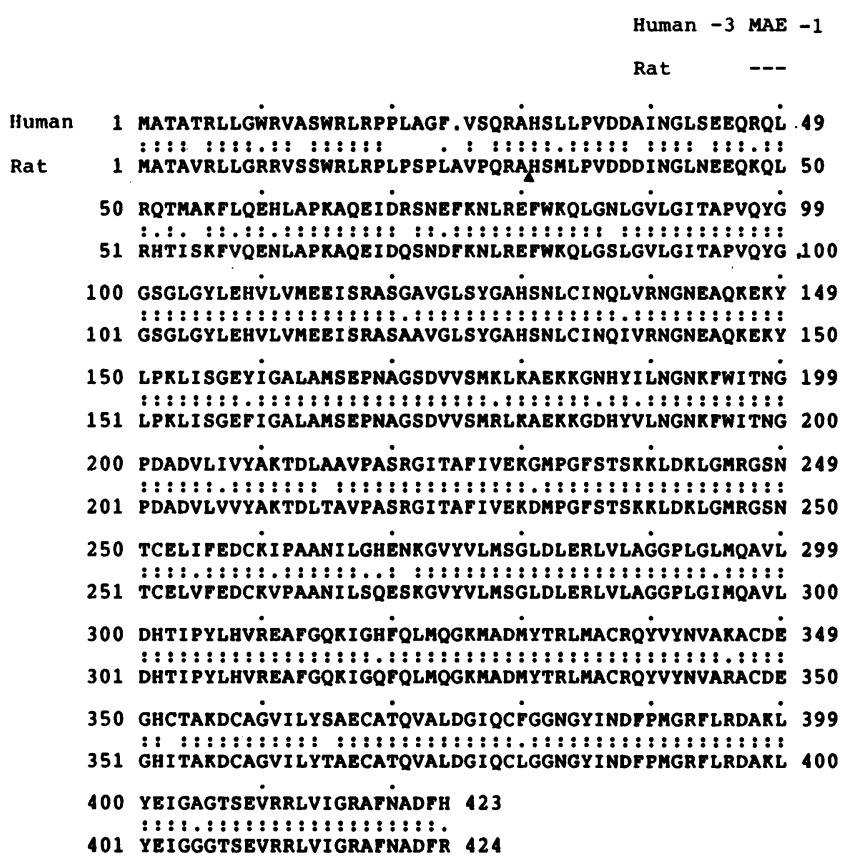

Figure 4. Comparison of the amino acid sequences of human and rat pIVD. Two dots between the sequences indicate perfect matches of amino acids. The amino acid pairs with one dot indicate those of conservative substitutions. The arrowhead indicates the cleavage site between the leader peptide and the mature protein of rat IVD.

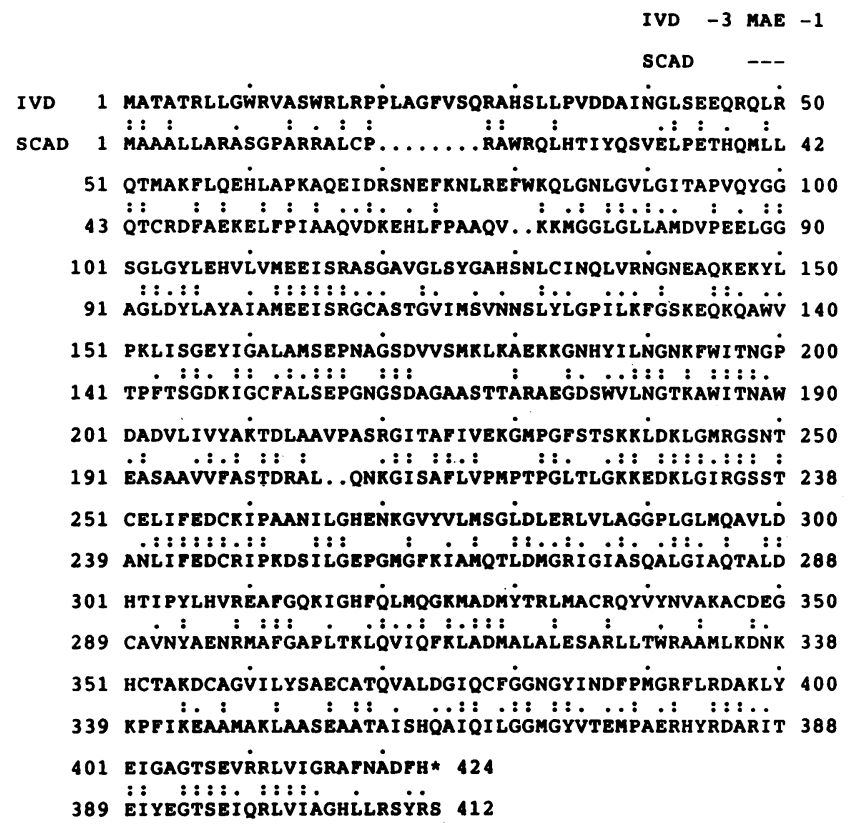

Figure 5. Comparison of the amino acid sequences of human pIVD and pSCAD. Two dots between the sequences indicate perfect matches of amino acids. The amino acid pairs with one dot indicate those of conservative substitutions.

ber of consecutive identical residues shared by human pIVD and pSCAD is only six. This difference in the number of consecutive identical residues is undoubtedly related to the molecular basis for the difference in the immuno-crossreactivity. The same acyl-CoA dehydrogenases from two different mammalian species are immunologically crossreactive $(10,28,35$, 36 ), but two different acyl-CoA dehydrogenases from the same species are not $(5,7)$.

In the comparison of human pIVD to human PSCAD and pMCAD, the homology in the leader peptide domain and the early amino terminal region of the mature enzyme is considerably lower than in the remaining part of the mature enzyme domain. This tendency applies also to the comparison of human and rat IVDs. In spite of the variability in the leader peptide sequence, the basic features of leader sequence are maintained in all acyl-CoA dehydrogenases: they all have high basic residue content and low or nonexistent in acidic residues.

Currently, the x-ray crystallographic data of porcine MCAD at $3 \AA$ are available (37). However, they only provide information on the backbone structure. Information on amino acid side-chains and the substrate-binding site is still unavailable. Thus, various enzymatic functions cannot be accurately assigned to individual amino acids or regions at present. In the mature peptide region, the sequences of human IVD, SCAD, and MCAD (data not shown) are more or less homologous throughout from the amino- to the carboxy-termini as in the case of rat acyl-CoA dehydrogenases (3). Thus, the domain structure of acyl-CoA dehydrogenases is not clear. The structural basis for the acyl-CoA dehydrogenase functions has been discussed in detail previously $(3,38)$.

At the mRNA level, the presence of three human pIVD mRNA was unexpected. Only a single mRNA species has been detected for other human and rat acyl-CoA dehydrogenases (3, $28,29)$. The nature of the two bands with larger molecular size 


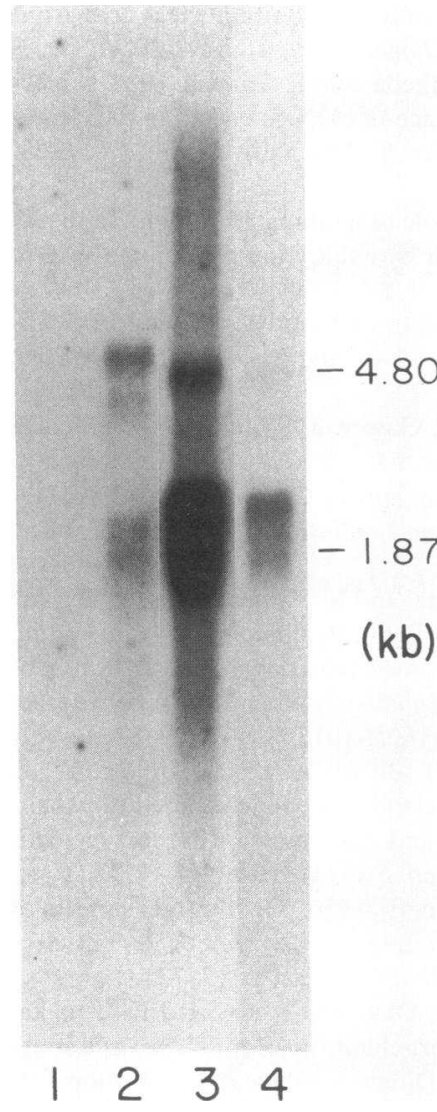

Figure 6. Northern blot analysis of mRNAs encoding human and rat $I V D$. The filter was hybridized with ${ }^{32} \mathrm{P}$-labeled insert of rIVD-A3, a fulllength clone (15). Lane 1,8 $\mathrm{mg}$ of human liver poly $(\mathrm{A})^{-}$ RNA; lane $2,10 \mu \mathrm{g}$ of human liver poly(A) ${ }^{+}$RNA; lane 3,10 $\mu \mathrm{g}$ of rat liver poly(A) ${ }^{+}$RNA. Lane 4 shows the short exposure $(5 \mathrm{~h})$ of lane 3 , which was exposed to the film for $36 \mathrm{~h}$.

(4.6 and $3.8 \mathrm{~kb}$ ) is currently unknown. The molecular size differences of the three mRNA species is too large to be accounted for by the small size differences of multiple mRNA species with different promoters in the $5^{\prime}$-noncoding region, such as those for acetyl-CoA carboxylase in rat liver and mammary gland (39). There are several possible causes for the multiple human pIVD mRNA species. The larger bands may represent RNA of nuclear origin or may be due to alternative or incomplete splicing of the mRNA. At present, the possibility of alternative polyadenylation sites is unlikely, since we have found in the $3^{\prime}$-noncoding region no such alternative sites, nor the sequence that may possibly cause structural exclusion of the polyadenylation signal at position 1,846.

The blot analysis of mRNA in cultured fibroblasts from patients with IVA provided useful information for the study of the molecular basis of various variant types. The size of variant $1,2,3$, and $5 \mathrm{mRNAs}$ were all similar, suggesting that mutation in all of them involves either a point mutation or a small deletion. The present data further support the hypothesis that the mutation in variant 1 is a point mutation. This variant is most commonly observed among the five variant types. 7 of 15 IVA cell lines so far studied were variant 1 homozygotes and two others were compound heterozygotes for variants 1 and 2 (10). The point mutation in different variant 1 cell lines may be heterogenous. Since accurate determination of the size of mRNA by electrophoretic analysis is difficult, distinction of point mutations or small deletions in variants 2 and 3 was not possible at present. The detection of normal-sized mRNA in variant 5 cells was unexpected. These data are useful for the further study of the mutation in this variant. The mutation in variant 5 may also be a small deletion or a point mutation in

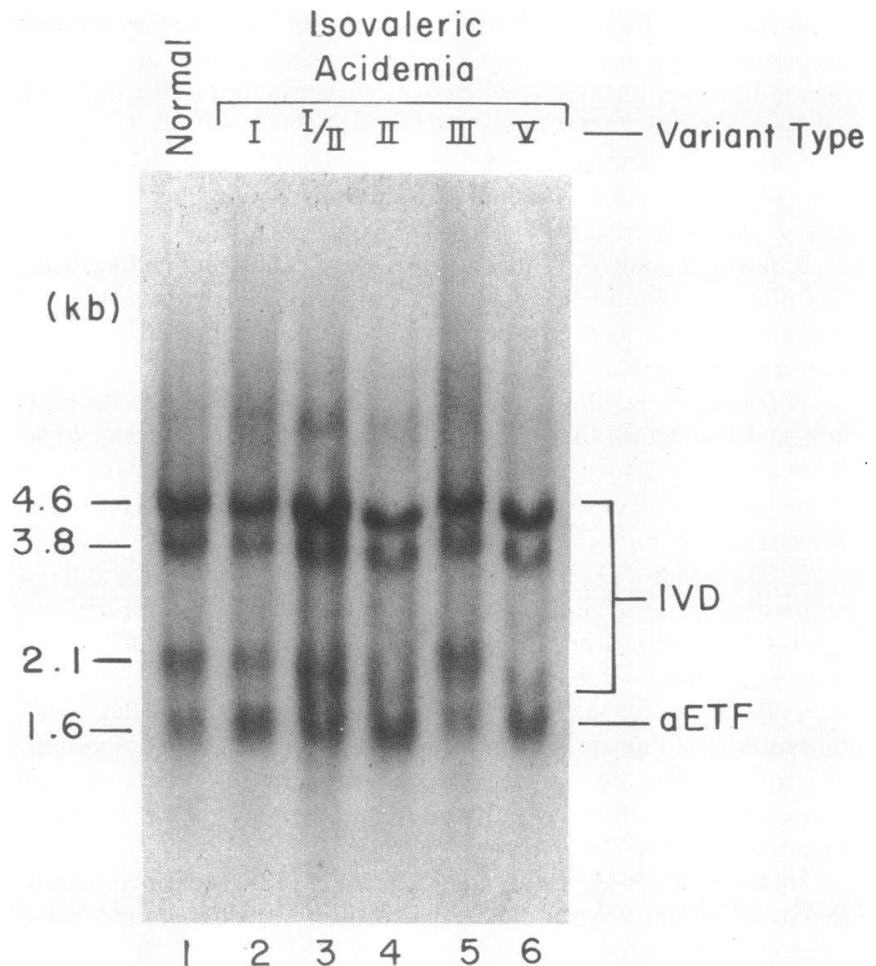

Figure 7. Northern blot analysis of mRNAs isolated from IVD fibroblasts. The filter was first hybridized with radiolabeled insert of hpIVD-8. After the filter was exposed to an $x$-ray film and thoroughly stripped of the human pIVD probe, a radiolabeled $\alpha$-ETF cDNA (E9f) (23) was hybridized to the same filter. The two resulting $\mathrm{x}$-ray films were accurately overlaid. Lane 1 , normal control fibroblast; lane 2, IVA variant 1 ; lane 3 , an IVA compound heterozygote (IVA variant $1 \times$ variant 2 ); lane 4 , IVA variant 2 ; lane 5 , IVA variant 3; lane 6, IVA variant 5. $10 \mu \mathrm{g}$ of poly(A) ${ }^{+}$RNA were applied to each lane.

the very early region in the gene, causing a frameshift or premature termination codon. Currently, experiments to identify these different types of mutations using the polymerase chain reaction are in progress in our laboratory to elucidate various types of mutations of the IVD gene.

\section{Acknowledgments}

We thank Ms. Connie Woznick for preparing this manuscript. This work was supported by grants from the National Institutes of Health (DK-17543) and the March of Dimes (1-378).

\section{References}

1. Tanaka, K., M. A. Budd, M. L. Efron, and K. J. Isselbacher. 1966. Isovaleric acidemia: a new genetic defect of leucine metabolism. Proc. Natl. Acad. Sci. USA. 56:236-242.

2. Rhead, W. J., and K. Tanaka. 1980. Demonstration of a specific mitochondrial isovaleryl-CoA dehydrogenase deficiency in fibroblasts from patients with isovaleric acidemia. Proc. Natl. Acad. Sci. USA. 77:580-583.

3. Matsubara, Y., Y. Indo, E. Naito, H. Ozasa, R. Glassberg, J. Vockley, Y. Ikeda, J. Kraus, and K. Tanaka. 1989. Molecular cloning and nucleotide sequence of cDNAs encoding the precursors of rat long chain acyl-coenzyme $A$, short chain acyl coenzyme $A$ and isovalerylcoenzyme A dehydrogenases: sequence homology of four enzymes of the Acyl-CoA dehydrogenase family. J. Biol. Chem. 264:1632116331. 
4. Ikeda, Y., C. Dabrowski, and K. Tanaka. 1983. Separation and properties of five distinct acyl-CoA dehydrogenases from rat liver mitochondria: identification of a new 2-methyl branched chain acyl-CoA dehydrogenase. J. Biol. Chem. 258:1066-1076.

5. Ikeda, Y., and K. Tanaka. 1983. Purification and characterization of isovaleryl coenzyme A dehydrogenase from rat liver mitochondria. J. Biol. Chem. 258:1077-1085.

6. Ikeda, Y., and K. Tanaka. 1983. Purification and characterization of 2-methyl-branched chain acyl coenzyme A dehydrogenase, an enzyme involved in the isoleucine and valine metabolism, from rat liver mitochondria. J. Biol. Chem. 258:9477-9487.

7. Ikeda, Y., K. Okamura-Ikeda, and K. Tanaka. 1985. Purification and characterization of short-chain, medium-chain, and longchain acyl-CoA dehydrogenases from rat liver mitochondria: isolation of the holo- and apoenzymes and conversion of the apoenzyme to the holoenzyme. J. Biol. Chem. 260:1311-1325.

8. Finocchiaro, G., M. Ito, and K. Tanaka. 1987. Purification and properties of short chain acyl-CoA, medium chain acyl-CoA, and isovaleryl-CoA dehydrogenases from human liver. J. Biol. Chem. 262:7982-7989.

9. Ikeda, Y., S. M. Keese, W. A. Fenton, and K. Tanaka. 1987. Biosynthesis of four rat liver mitochondrial acyl-CoA dehydrogenases: in vitro synthesis, import into mitochondria, and processing of their precursors in a cell-free system and in cultured cells. Arch. Biochem. Biophys. 252:662-674.

10. Ikeda, Y., S. M. Keese, and K. Tanaka. 1985. Molecular heterogeneity of variant isovaleryl-CoA dehydrogenase from cultured isovaleric acidemia fibroblasts. Proc. Natl. Acad. Sci. USA. 82:7081-7085.

11. Budd, M. A., K. Tanaka, L. B. Holmes, M. L. Efron, J. D. Crawford, and K. J. Isselbacher. 1967. Isovaleric acidemia: clinical features of a new genetic defect of leucine metabolism. N. Engl. J. Med. 277:321-327.

12. Tanaka, K., and L. E. Rosenberg. 1983. Disorders of branched chain amino acid and organic acid metabolism. In The Metabolic Basis of Inherited Disease. J. B. Stanbury, J. B. Wyngaarden, D. S. Fredrickson, J. L. Goldstein, M. S. Brown, editors. 5th edition. McGraw-Hill Inc., New York. 440-473.

13. Hyman, D. B., and K. Tanaka. 1986. Isovaleryl-CoA dehydrogenase activity in isovaleric acidemia fibroblasts using an improved tritium release assay. Pediatr. Res. 20:59-61.

14. Dubiel, B., C. Dabrowski, R. Wetts, and K. Tanaka. 1983. Complementation studies of isovaleric acidemia and glutaric aciduria type II using cultured skin fibroblasts. J. Clin. Invest. 72:1543-1552.

15. Kraus, J. P., Y. Matsubara, D. Barton, T. L. Yang-Feng, R. Glassberg, M. Ito, Y. Ikeda, J. Mole, U. Francke, and K. Tanaka. 1987. Isolation of cDNA clones coding for rat isovaleryl-CoA dehydrogenase and assignment of the gene to human chromosome 15. Genomics. 1:264-269.

16. Feinberg, A. P., and B. Vogelstein. 1983. A technique for radiolabeling DNA restriction endonuclease fragments to high specific activity. Anal. Biochem. 132:6-13.

17. Chen, E. Y., and P. H. Seeburg. 1985. Supercoil sequencing: a fast and simple method for sequencing plasmid DNA. DNA (NY) 4:165-170.

18. Sanger, F., S. Nicklen, and A. R. Coulson. 1977. DNA sequencing with chain-terminating inhibitors. Proc. Natl. Acad. Sci. USA. 74:5463-5467.

19. Benson, W. D., and R. W. Davis. 1977. Screening $\lambda$ gt recombinant clones by hybridization to single plaques in situ. Science (Wash DC). 196:180-182.

20. Maniatis, T., E. F. Fritsch, and J. Sambrook. 1982. Molecular Cloning: A Laboratory Manual. Cold Spring Harbor Laboratory, Cold Spring Harbor, NY. 1-545.

21. Devereux, J., P. Haeberli, and O. Smithies. 1984. A comprehensive set of sequence analysis programs for the VAX. Nucleic Acids Res. 12:387-395.

22. Chirgwin, J. M., A. E. Przybyla, R. J. MacDonald, and W. J.
Rutter. 1979. Isolation of biologically active ribonucleic acid from sources enriched in ribonuclease. Biochemistry. 18:5294-5299.

23. Finocchiaro, G., M. Ito, Y. Ikeda, and K. Tanaka. 1988. Molecular cloning and nucleotide sequence of cDNAs encoding the $\alpha$-subunit of human electron transfer flavoprotein. J. Biol. Chem. 263:15773-15780.

24. Kozak, M. 1981. Possible role of flanking nucleotides in recognition of the AUG initiator codon by eukaryotic ribosomes. Nucleic Acids Res. 9:5233-5252.

25. Kozak, M. 1984. Compilation and analysis of sequences upstream from the translational start site in eukaryotic mRNAs. Nucleic Acids.Res. 12:857-872.

26. Hay, R., P. Böhni, and S. Gasser. 1984. How mitochondria import proteins. Biochim. Biophys. Acta. 779:65-87.

27. von Heijne, G., J. Steppuhn, and R. G. Herrmann. 1989. Domain structure of mitochondrial and chloroplast targeting peptides. Eur. J. Biochem. 180:535-545.

28. Naito, E., H. Ozasa, Y. Ikeda, and K. Tanaka. 1989. Molecular cloning and nucleotide sequence of complementary DNAs encoding human short chain acyl-coenzyme A dehydrogenase and the study of the molecular basis of human short chain acyl-coenzyme A dehydrogenase deficiency. J. Clin. Invest. 83:1605-1613.

29. Kelly, D. P., J.-J. Kim, J. J. Billadello, B. E. Hainline, T. W. Chu, and A. W. Strauss. 1987. Nucleotide sequence of medium-chain acyl-CoA dehydrogenase mRNA and its expression in enzyme-deficient human tissue. Proc. Natl. Acad. Sci. USA. 84:4068-4072.

30. Crane, F. L., and H. Beinert. 1956. On the mechanism of dehydrogenation of fatty acyl derivatives of coenzyme A. II. The electron-transferring flavoprotein. J. Biol. Chem. 218:717-731.

31. Ikeda, Y., D. G. Hine, K. Okamura-Ikeda, and K. Tanaka. 1985. Mechanism of action of short-chain, medium-chain, and longchain acyl-CoA dehydrogenases. Direct evidence for carbanion formation as an intermediate step using enzyme-catalyzed C-2 proton/ deuteron exchange in the absence of C-3 exchange. J. Biol. Chem. 260:1326-1337.

32. Tanaka, K., Y. Ikeda, Y. Matsubara, and D. B. Hyman. 1987. Molecular basis of isovaleric acidemia and medium-chain acyl-CoA dehydrogenase deficiency. Enzyme (Basel). 38:91-107.

33. Matsubara, Y., J. P. Kraus, T. L. Yang-Feng, U. Francke, L. E. Rosenberg, and K. Tanaka. 1986. Molecular cloning of cDNAs encoding rat and human medium-chain acyl-CoA dehydrogenase and assignment of the gene to human chromosome 1. Proc. Natl. Acad. Sci. USA. 83:6543-6547.

34. Matsubara, Y., J. P. Kraus, H. Ozasa, R. Glassberg, G. Finocchiaro, Y. Ikeda, J. Mole, L. E. Rosenberg, and K. Tanaka. 1987. Molecular cloning and nucleotide sequence of cDNA encoding the entire precursor of rat liver medium chain acyl coenzyme A dehydrogenase. J. Biol. Chem. 262:10104-10108.

35. Ikeda, Y., and K. Tanaka. 1987. Immunoprecipitation and electrophoretic analysis of four human acyl-CoA dehydrogenases and electron transfer flavoprotein using antibodies raised against the corresponding rat enzymes. Biochem. Med. 37:329-334.

36. Ikeda, Y., D. E. Hale, S. M. Keese, P. M. Coates, and K. Tanaka. 1986. Biosynthesis of variant medium chain acyl-CoA dehydrogenase in cultured fibroblasts from patients with medium chain acyl-CoA dehydrogenase deficiency. Pediatr. Res. 20:843-847.

37. Kim, J.-J. P., and J. Wu. 1988. Structure of the medium-chain acyl-CoA dehydrogenase from pig liver mitochondrial at 3-Å resolution. Proc. Natl. Acad. Sci. USA. 85:6677-6681.

38. Tanaka, K., Y. Matsubara, Y. Indo, E. Naito, J. Kraus, and H. Ozasa. 1990. The acyl-CoA dehydrogenase family: homology and divergence of primary sequence of four acyl-CoA dehydrogenases, and consideration of their functional significance. In Fatty Acid Oxidation: Clinical, Biochemical and Molecular Aspects. K. Tanaka and P. M. Coates, editors. Alan R. Liss, Inc., New York. 577-598.

39. López-Casillas, F., and K.-H. Kim. 1989. Heterogeneity at the $5^{\prime}$ end of rat acetyl-coenzyme A carboxylase mRNA. J. Biol. Chem. 264:7176-7184. 